Ergod. Th. \& Dynam. Sys. (1983), 4, 147-163

Printed in Great Britain

\title{
Lorenz knots are prime
}

\author{
R. F. WILLIAMS \\ Mathematics Department, Northwestern University, Evanston, IL 60201, USA
}

(Received 4 August 1982 and revised 30 June 1983)

In celebration of Ralph P. Boas's seventieth birthday.

\begin{abstract}
Lorenz knots are the periodic orbits of a certain geometrically defined differential equation in $\mathbb{R}^{3}$. This is called the 'geometric Lorenz attractor' as it is only conjecturally the real Lorenz attractor. These knots have been studied by the author and Joan Birman via a 'knot-holder', i.e. a certain branched two-manifold $H$. To show such knots are prime we suppose the contrary which implies the existence of a splitting sphere, $S^{2}$. The technique of the proof is to study the intersection $S^{2} \cap H$. A novelty here is that $S^{2} \cap H$ is likewise branched.
\end{abstract}

\section{Introduction}

In two previous papers [1], [2], Joan Birman and the author have begun a study of the periodic orbits of certain flows $\phi_{t}$ on a 3-manifold $M^{3}$, often $\mathbb{R}^{3}$ or $S^{3}$. Our attempt is to bring parts of knot theory to bear, as in particular a periodic orbit of $\phi_{t}$ is a 1-sphere embedded in $M^{3}$ and thus a knot. We conjectured the theorem of the title of this paper in [1].

We are especially interested in how the various periodic orbits of a single flow (or ordinary differential equation) are related to one another. The principal tool introduced in [1], [2] was the 'knot holder' for $\phi_{t}$, i.e. a 2-dimensional branched manifold $H \subset M^{3}$ and semi flow $\bar{\phi}_{t}$, defined for $t \geq 0$ on $H$. The idea is that the family of all periodic orbits of the original flow $\phi_{t}$ on $M^{3}$ are pushed down into the 2-dimensional setting of $\bar{\phi}_{t}$ on $H$. The isotopy properties of the orbits are preserved by this process. The overall goal is to obtain global theorems as to which families of knots occur.

The reader is referred to [1], [2] for material on knot holders; we summarize some of this here.

A knot holder $H$, consists of finitely many 'joining' and 'splitting' charts put together by sewing each bottom to exactly one top and vice versa. The joining chart has the defect that flow lines come together along the branch set $\beta$ and thus $\bar{\phi}_{t}$ is not well defined for $t<0$. Likewise the flow leaves splitting charts at the bottom and hence $\bar{\phi}_{t}$ is not defined for some $t>0$. The interesting orbits are those which remain in $H$ for all $t \geq 0$. A certain symbolic dynamics of knot holders was worked out in $[2, \S 5]$ and will be used in this paper. 


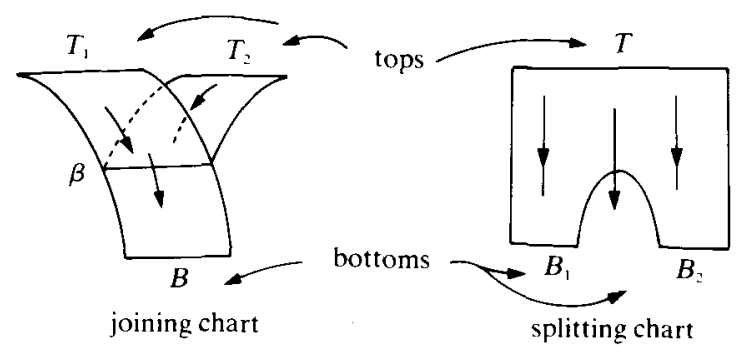

The simplest knot holders consist of just 2 charts, one of each kind. There is a 2 parameter family of these and we will adopt the notation $L(u, v), u, v \in \mathbb{Z}$. This involves a convention on twists;

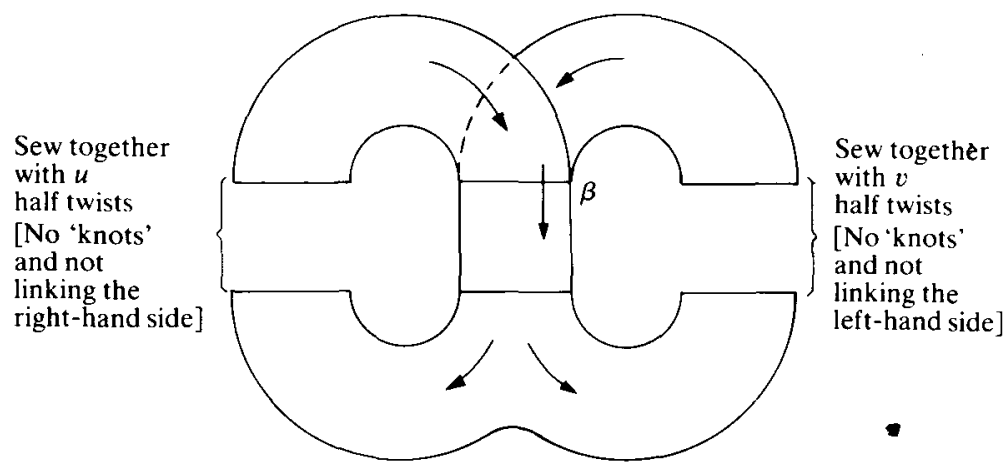

The knot holder $L(u, v)$

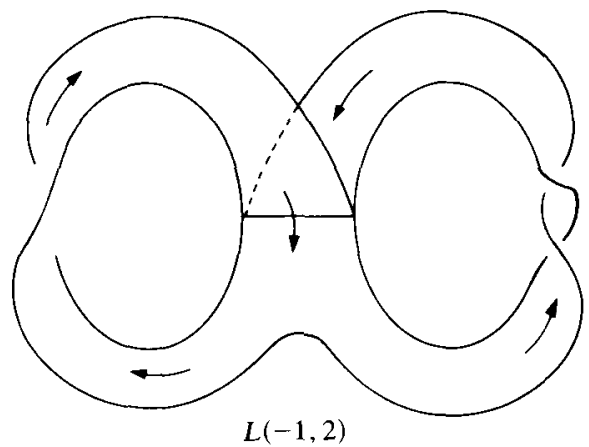

ours is that left-handed twists are positive, right-handed negative, as in our example. In addition there is the symmetry $L(u, v)=L(v, u)$ via rotation of $\pi$ about the vertical; also the mirror reflection $\bar{L}(u, v)$ of $L(u, v)$. We say $L(u, v)$ is left-handed, $\bar{L}(u, v)$ right-handed. The Loren $z$ knot holder is $L(0,0)$, and derives its name from the 'Lorenz attractor'; in effect, $L(0,0)$ is the 'preturbulent' version of the differential equations

$$
\begin{aligned}
& \dot{x}=-\sigma x+y, \\
& \dot{y}=R x-y \quad-x z, \\
& \dot{z}=\quad-b z+x y,
\end{aligned}
$$


where $\sigma=10, b=\frac{8}{3}$ and $R$ is about 16 . That is to say, by perturbation methods one finds that the periodic solutions to the Lorenz equations are exactly those of $L(0,0)$. Though no one has succeeded in giving an analytic derivation of the nature of the 'Lorenz attractor', $R=28$ or nearby, most people believe the 'model' as described by Guckenheimer [3], [4] and Williams [5] corresponds to reality. This model is essentially that given by $L(0,0)$, except that in any one of the attractors, certain of the periodic orbits of $L(0,0)$ would be missing. It is to be hoped that the techniques developed here will aid in studying the more general problem of prime-factorization of knotted periodic orbits. In particular, the machinery developed here proves slightly more than the title:

Theorem. For any $v \geq 0$, the knots in $L(0, v)$ and $\bar{L}(0, v)$ are prime.

Example. For $u \neq 0, v \neq 0, L(u, v)$ and $\bar{L}(u, v)$ contain composite knots.

Definition. A knot $K$ is said to be prime provided it is not the connected sum of any 2 knots. $K \subset \mathbb{R}^{3}$ is said to be the connected sum of the knots $K_{1}$ and $K_{2}$ provided there is a smooth 2 -sphere $S^{2} \subset \mathbb{R}^{3}$ such that $S^{2}$ intersects $K$ transversally in exactly 2 points, say $K \cap S^{2}=\{a, b\}$, so that if $\alpha$ is an arc on $S^{2}$ joining $a$ to $b$, then $\alpha \cup \gamma_{i}$ is equivalent to $K_{i}$, where $\gamma_{1}=K \cap\left(\right.$ interior of $S^{2}$ ) and $\gamma_{2}=K \cap\left(\right.$ exterior of $S^{2}$ ).

We close the introduction with a brief discussion of the 'symbolic dynamics' of the knot holder $L(u, v)$. See [1] or [2] for more details. Note that each $L(u, v)$ has the homotopy type of the wedge of two one-spheres. We take the generators of the fundamental group to be $x$ on the left and $y$ on the right. Orient $x$ and $y$ in the direction of the flow, $\bar{\phi}_{t}$. Then the periodic orbits of $\phi_{t}$ correspond 1-to- 1 to the cyclic permutation classes of positive words in $x$ and $y$. See [1] or [2]. Similarly for other knot holders, except that more than 2 symbols may be required. We use the $x y$ word of periodic orbits below beginning in $\S 3$.

I would like to thank several people for helpful conversations, in particular Joan Birman and the referee; the latter found an error in my earlier version in addition to making several good suggestions. Some of the work on this paper was carried out while the author was at Boston University.

\section{Examples with composite knots}

Example. In the knot holder $L(u, v)$ or $\bar{L}(u, v)$ with $u \neq 0, v \neq 0$, the knots corresponding to $x^{n} y^{m}$ are all composite for $n, m$ sufficiently large.

Proof. In fact $n \geq 4, m \geq 4$ always suffice. We will only give these examples in certain cases:

(a) $x^{2} y^{2}$ in $L(3,3)$ and $L(2,2)$
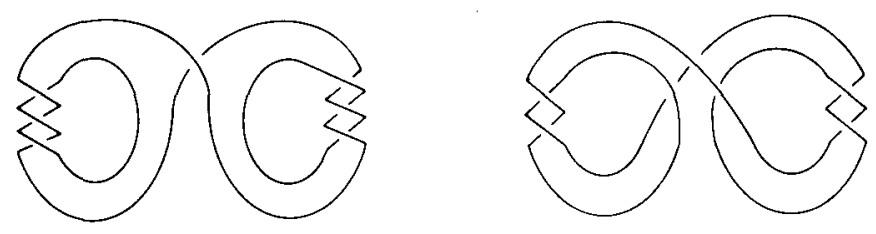
In both cases we get the granny knot $=$ the direct sum of two left handed trefoil knots. In $L(3,-3) x^{2} y^{2}$ is the square knot, i.e. the direct sum of a left handed and a right handed trefoil knot. Similarly for $|u|,|v| \geq 3 x^{2} y^{2}$ is composite in $L(u, v)$.

(b) In $L(-1,-1) x^{4} y^{4}$ is the right handed granny knot.

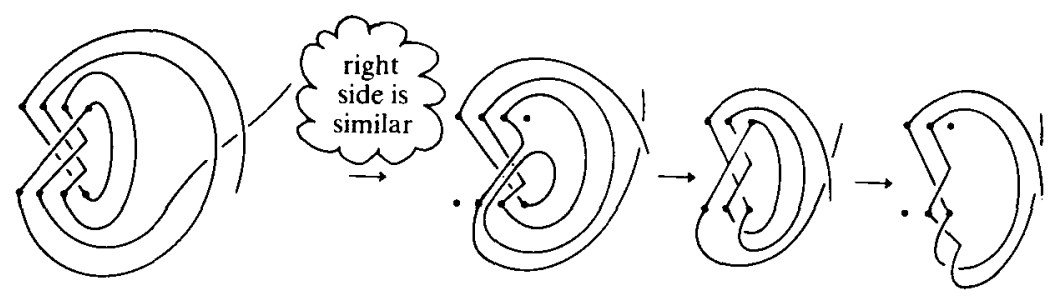

(c) In $L(1,1) x^{3} y^{3}$ is the granny knot.

(d) In $L(0,-1) x^{2} y^{4} x^{2} y$ is the square knot.
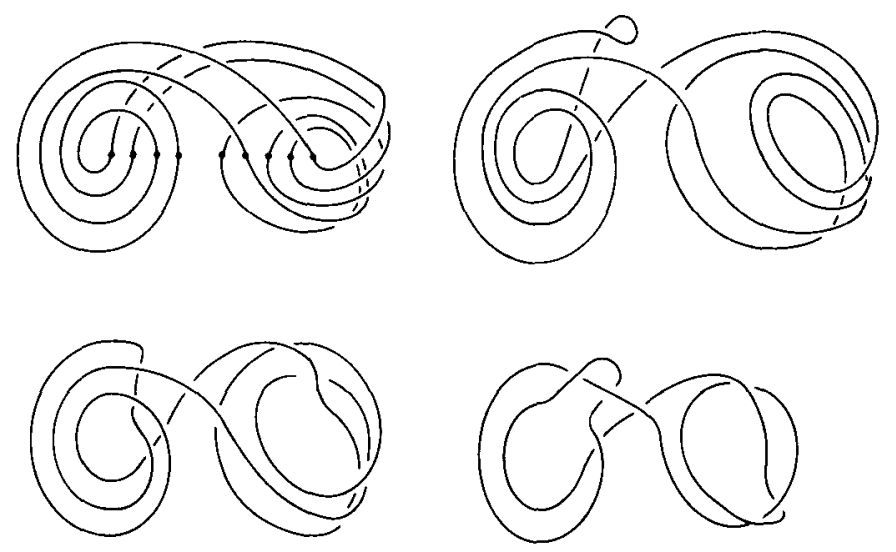

\section{Basic procedure}

The proof proceeds as follows: we assume we have a composite knot $K$ lying in our knot holder $H$ and satisfying the definition above. We then proceed to put the two-sphere $S^{2}$ in a nice position relative to $H$ and $K$. For the time being we deal with a general knot holder $H$. First we may assume $S^{2} \cap H$ is transverse at each point. (Beginning at the branch line, then proceeding to the two dimensional part, as usual.) Next we smooth out the intersection $S^{2} \cap H$. We may assume $S^{2} \cap H$ consists of a branched 1-manifold, the branch occurring only at the branch line of $L$. This is standard.

Next, we know that $S^{2} \cap H$ is roughly along the flow lines of $\bar{\phi}_{t}$, since it intersects the orbit $K$ in at most 2 points. Thus by small pushes, we may assume that $S^{2} \cap H$ is along the flow lines of $\bar{\phi}_{t}$ except at short stretches where it either doubles back, or moves transversely from one side to the other.

LEMMA 1.1. By a small push, we can remove any doubling back arc that occurs just below the branch line. 
Proof. This is a picture:
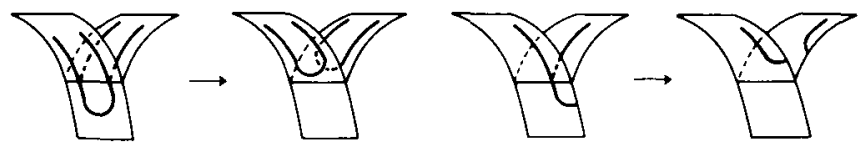

The heavy line, here and below, is $S^{2} \cap H$.

Lemma 1.2. ('No double entry lemma'.) At no point does $S^{2} \cap H$ cut across two adjacent strands of the knot as in the figure:
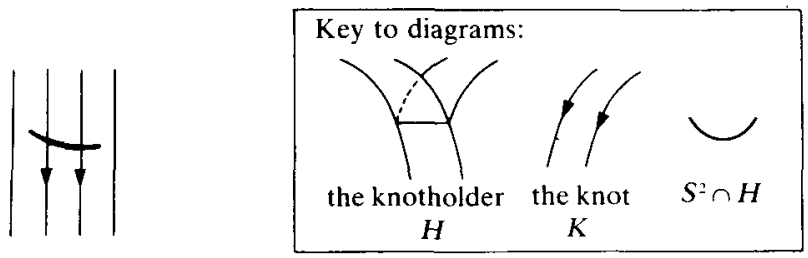

Proof. In this case the knot $K$ would be passing from one side of the sphere to the other, both times, which is absurd.

\section{Segments in $S^{2} \cap H$}

Proposition. We may deform $S^{2}$ so that $S^{2} \cap H$ lies entirely along flow lines of $\bar{\phi}_{t}$ with the exception of $U$-joints, edge joints, trivial closed curves and short diagonal stretches. Each of these exceptional sets lies just above some branch line. Only arcs which connect 2 points of the branch set contain diagonal stretches.

This proposition is a summary of the following:

LEMMA 2.1. We may adjust $S^{2}$ such that $S^{2} \cap H$ consists of components which either (a) are trivial simple closed curves, i.e. those which intersect no branch line and bound disks; or (b) are the union of arcs which connect two points on the branch set union the boundary of $H$. These arcs are called segments of $S^{2} \cap H$ and consist of 4 types:

(1) almost straight arcs - except for possibly a short diagonal stretch immediately above a branch point, they lie on a flow line and connect two points of $\partial H \cup \beta$;

(2) $U$-joints - these lie immediately above a branch line and connect 2 of its points;

(3) edge joints - these lie immediately above a branch line and connect a point of this branch line to the boundary, $\partial H$;

(4) double edge joints - these connect 2 points on opposite sides of $\partial H$ and are essentially parallel to the branch set.

Remark about the proof. A good image (suggested by the referee) is to 'comb' hair straight which is fixed at the bottom. You comb straight down almost to the bottom and leave all the 'tangles' at the bottom. The bottom is of course the branch line. The tangles include all of the exceptional sets. If an $\operatorname{arc} \alpha$ of $S^{2} \cap H$ intersects the branch line at a point $a$, let $\beta$ be the flow line leaving the branch set at $a$. Then one pushes $\alpha$ so as to agree with $\beta$ down until one is near the branch set, where we must allow it to do its own thing. 
At times it is preferable to comb upwards, especially after the initial combing. That is we start at the bottom and straighten an intersection arc of $S^{2} \cap H$ so as to remain on a flow line. In upward combing, only the almost straight arcs are changed; the $U$-joints, edge joints and double edge joints are left unchanged. The advantage of upward combing is that one can comb a loop off of a disk edge. This is because the periodic orbit at a disk edge is a repellor, so, proceeding backwards, it acts as an attractor. See 5.1 below.

Proof. If there is any doubling back, one pushes the intersection so as to decrease a max or increase a min until one arrives near a point of the boundary or a branch point. One simply pushes an offending minimum up through a branch line, via lemma 1. Maxima come in 2 types: when they arrive at a branch line they become $U$-joints or edge joints. Maxima could be pushed into the boundary, since the flow $\bar{\phi}_{t}$ leaves $H$ along local maxima of $\partial H$, as in the figure

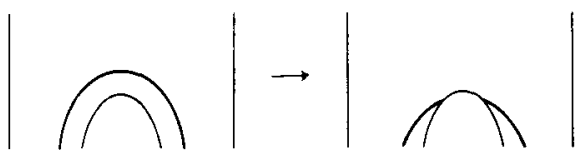

These create 2 'edge maxima', which can be pushed downward until they arrive at a branch line; they would then be edge-joints.

Now if we have an arc which begins at a boundary or branch point $p$, and proceeds upward to a branch point, we may deform it to lie along the flow line through $p$, with no trouble until we arrive at the upper branch line.

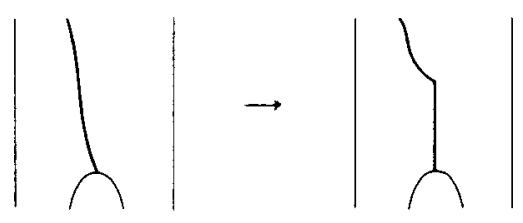

This works just as well at a branch line, since there is at most one point of the knot on this part of $S^{2} \cap H$. We might have to leave, however, a diagonal stretch immediately above the branch line, to avoid an infinite loop. But this is O.K.

\section{Minimality assumption}

In order to complete the proof, we assume that we are in a minimal situation: to wit

(a) the number of symbols in our knot $K$ is the smallest of any composite knot carried by $K$, and

(b) the number of segments (see $\S 2$ ) in the intersection $S^{2} \cap H$ is the least possible integer relative to the knot $K$, and

(c) the number of branch points of the intersection $S^{2} \cap H$ is least possible relative to $(a)$ and $(b)$.

We then proceed to a contradiction by showing the intersection is not minimal. 


\section{U-strings}

Definition. By a $U$-string at a branch line $\beta$ is meant a finite sequence $U_{1}, U_{2}, \ldots, U_{n}$ of $U$-joints at $\beta$, such that $U_{i} \cap U_{i+1}$ is an end point of both $U_{i}$ and $U_{i+1}$, $i=1, \ldots, n-1$.

We also allow either $U_{1}$ or $U_{n}$ or both to be an edge joint. We allow as a degenerate case, a 'double edgejoint', i.e. a segment of $S^{2} \cap H$ passing horizontally from one edge to another. This would be just above, and essentially parallel to, a branch line.

LEMMA 4.1. U-strings do not double back.

Proof. Assume the contrary and look at an extreme point of the $U$-string:

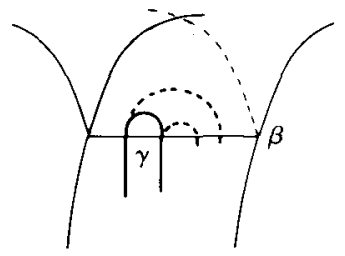

Then there is a segment $\gamma$ of $\beta$ cut off by $U$-joints on both sides.

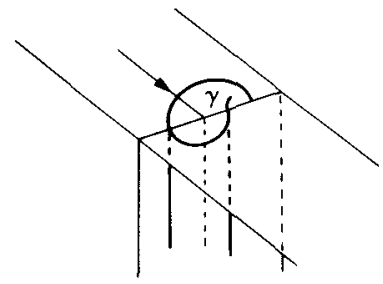

First we claim that at most one of the $2 U$-joints is pierced by our knot. In fact one sees that any such piercing is toward a single side of $S^{2}$, the $\gamma$-side.

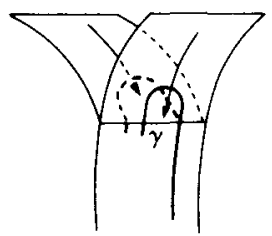

(That is to say, the side of $S^{2}$ containing $\gamma$, or at least that part of it near the boundary of $\gamma$.) But $K$ pierces $S^{2}$ only twice, once entering the $\gamma$-side, and once leaving.

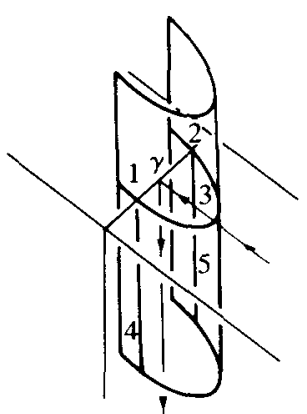


Now look at the $U$-joint cutting off the arc $\gamma$. We draw the case in which this $U$-joint is pierced by the knot $K$. The other case is similar. Thus near $\gamma$ we have a 'tunnel' formed by $S^{2}$, which we can deform as follows:

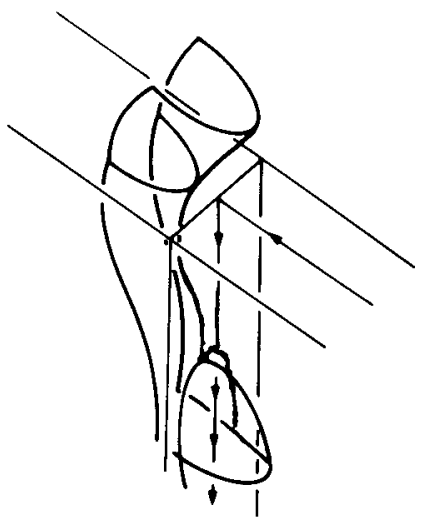

Note first that 2 branch points have been removed from $S^{2} \cap H$. Next the two newly introduced local max and min look like so:

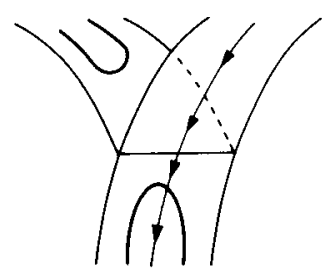

But since the lower ones can be pushed down we need to see how they end up. In one case we get 2 edge loops.

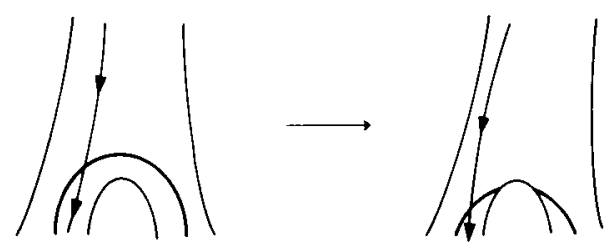

But in any case we get at most 2 segments. The upper edge was formed from joining segments labelled 1 and 2 in our diagram. These could be part of the same segment, a $U$-joint. In this case, we have created

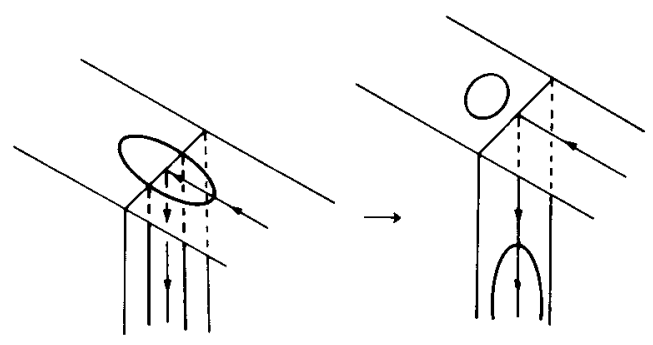


a new trivial loop, which doesn't count at all. In either case we have a reduction in the number of segments by one. Finally, the original $U$-joint labelled 3 has been removed, for a total of at least two segments less. This contradicts the minimality and proves our lemma.

LEMMA 4.2. Each segment of a $U$-string, including end joints if any, has an arc of the knot $K$ on its free side, 'guarding' it.

Proof. The deformation indicated in the proof of the previous lemma is possible, unless an $\operatorname{arc}$ of $K$ 'guards' the appropriate $U$-joint:

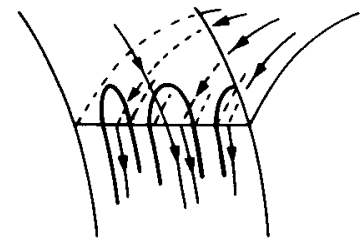

guarded $U \cdot$ joints

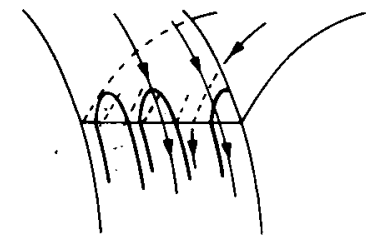

unguarded

Note that an arc of $K$ passing through a $U$-joint or an edge-joint is not enough to 'guard it'. The edge-joint case:

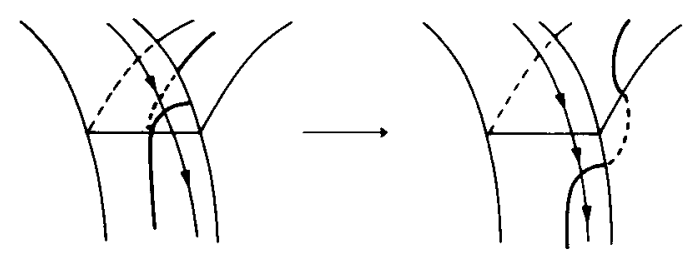

We have a reduction of at least one in segments.

\section{There is an edge joint at a disk edge}

To prove this proposition we first rule out the trivial case in which $S^{2}$ does not intersect the branch line. For then $S^{2} \cap H$ lies in a disk lying in $H$. Some component of $S^{2} \cap H$ hits our knot; this component is a 1 -sphere and bounds a disk lying in $H$. Since our knot enters this disk, it also leaves it, and these are the intersections of $S^{2}$ with the knot. Thus one of the components of our composite knot lies in a disk which is absurd.

At this point we need to begin using our assumption that $H=L(0, v), v \geq 0$.

So we begin at a point of $S^{2}$ on the branch line and proceed upward from it in our search. We may be forced to use $U$-strings to continue our upward progress, and in this case we must be careful. But even without using $U$-strings, we may $a$ priori get caught in an infinite loop. Thus we must study the possible loops, or simple closed curves in $S^{2} \cap H$; we have already dealt with the trivial loops - those not intersecting the branch line. We now deal with the second simplest type.

LEMMA 5.1. Each non-trivial loop $\Lambda$ of $S^{2} \cap H$ contains a U-string.

Proof. Suppose $\Lambda$ is a loop of $S^{2} \cap H$ which intersects the branch line and contains no $U$-string. Then $\Lambda$ is the union of short diagonal stretches plus more important 
sub-intervals which lie along the flow lines. These latter are of two types, $x$-arcs which pass around the knot holder on the left (or $x$-side) and $y$-arcs which pass around on the right. But the tangent bundle to $\Lambda$ in $K$ must be untwisted because $\Lambda$ lies in $S^{2}$ where it has an untwisted tangent bundle. To complete the proof of 5.1 we need the formula in the following:

Lemma 5.2. If $\Lambda$ is a loop with no $U$-strings then the twist \# of $\Lambda=(\# x$-arcs $)+(\# y$-arcs $)(v / 2+1)-1$.

Proof. Here $v$ is the number of half twists in our knot-holder $H=L(0, v)$, which explains the contribution of $v / 2$ in the formula. The other part is well known - for example the curve $x y$ in case $v=0$ has twist \# $=1$.

Hence, as $\Lambda$ must have twist \#=0, there are two possibilities: (1) $\Lambda$ has only an $x$-arc, and (2) $v=0$ and $\Lambda$ has only a $y$-arc. As the two cases are perfectly similar we assume case (1) holds.

Next, note that if $\Lambda$ does not intersect our knot, it can be pushed into the hole at the disk edge. This reduces the number of segments and thus contradicts the minimality assumption. Now assume our orbit does intersect the knot. Then it must intersect it only along the diagonal stretch and only at one point, say $q$.

Let $a$ be the point of the branch set on the disk edge and let $b$ be the point of our loop on the branch set. Now proceeding upward on our knot from $q$ we arrive at a point $p$ on the branch set between $a$ and $b$. See the diagram. We claim $p$ is the leftmost point on the branch set, as otherwise the knot would either be spinning trivially and thus not minimal, or two distinct segments of the knot $K$ would be entering the left side at $[a, b]$. This last would contradict the no-double-entry lemma 1.2. Now comb the loop upward $(\$ 2)$ and we arrive at a loop which misses the knot entirely, reducing us to the earlier case above.
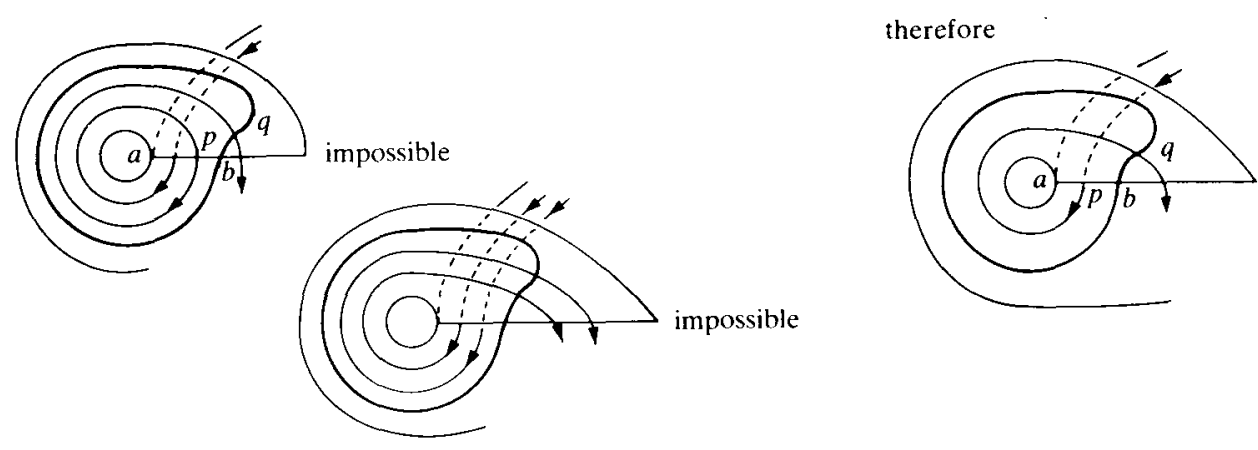

thus comb up
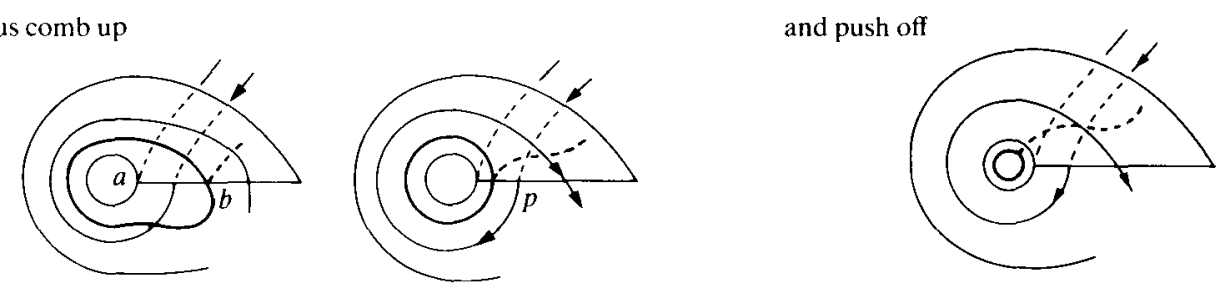

FIGURE A 
Thus we turn our attention to loops which contain a $U$-string. We need first to assess the contribution of the $U$-joints to the twist number of the normal bundle to a loop. Thus suppose we have 2 straight $\operatorname{arcs} \alpha, \beta$ connected by a $U$-joint chain. We take notation so that proceeding upward we have $\alpha$ first, then the $U$-joint chain $\gamma$, then $\beta$. Let $\gamma$ have $m$ segments.

(5.3) For the purpose of computing the contribution of $\gamma$ to the twisting number, we may assume it has either one cusp or none at all.

Proof. If $\gamma$ has more than one cusp, then let $\gamma_{0}$ be, say, the rightmost $U$-joint connecting 2 cusps. Now the operation of folding $\gamma_{0}$ down below the branch line reduces the number of cusps by 2 , and we claim this does not change $\gamma$ 's contribution to the twist number. (We don't claim this is a legitimate perturbation to $S^{2}$ as usually it is not. It serves only to compute the twist contribution.) See the accompanying figure.
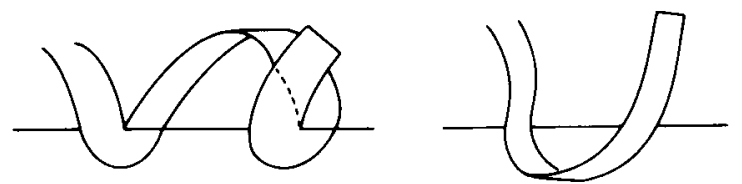

Thus we end up with either one or none at all.

(5.4) We need to develop some notation to monitor our path. Proceeding upward, we trace out a path with 3 kinds of segments: $x$-arcs, $y$-arcs and $U$-strings. We use $x$ 's and $y$ 's to monitor the first. We further subdivide the $U$-string paths as neutral, $\mathrm{N}$, in the case where it has an even number of cusps, and otherwise as $\mathrm{R}$ or $\mathrm{L}$ depending upon whether we pass along it to the right or left. Thus our path upwards is monitored by an ordered string of symbols such as $x \mathrm{NyL} x \mathrm{R} \ldots$. Of course if we trace out a loop $\Lambda$ only the cyclic order is significant so that $x \mathrm{~L} y \mathrm{~L} \approx y \mathrm{~L} x \mathrm{~L}$.

LEMMA 5.5. The $U$-joint $\gamma$ contributes $+\frac{1}{2}$ in the cases $\mathrm{L} y$ and $\mathrm{R} x$ and $-\frac{1}{2}$ in the cases $\mathrm{L} x$ and $\mathrm{R} y$.

Proof. See figure B.
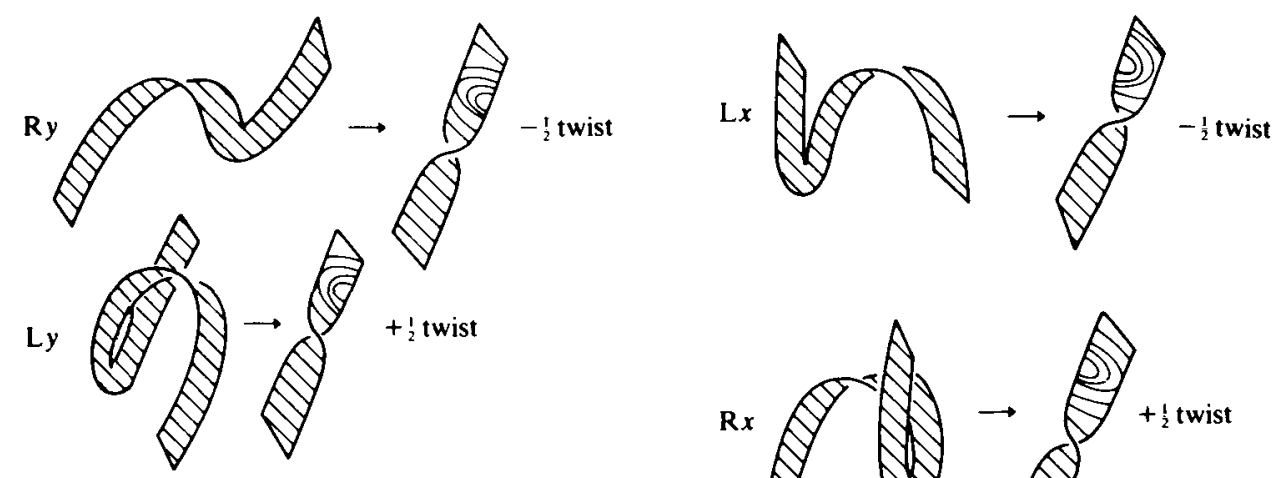

Figure B

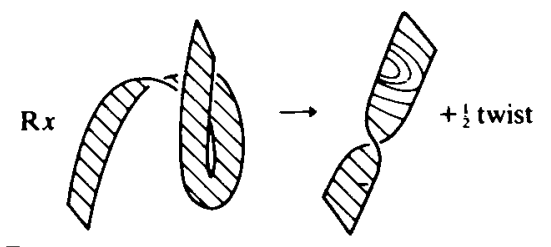


LEMMA 5.6. The twisting number of the normal bundle to $\Lambda$ is given by

$$
\# x-\operatorname{arcs}+(v / 2+1)(\# y \text {-arcs })-1+\frac{1}{2}(\# \mathrm{~L} y+\# \mathrm{R} x-\# \mathrm{~L} x-\# \mathrm{R} y) \text {. }
$$

The symbols in the parenthesis mean the number of occurrences of each case, as above.

Proof. This is just a combination of our previous formula 5.2 together with our consideration of cusps, above.

LEMMA 5.7. The only possible loops are those with the symbol

(1) $\mathrm{N} x$ for any $v \geq 0$;

(2) $\mathrm{Ny}$ for $v=0$;

(3) $\mathrm{R} y, v=1$;

(4) $\mathrm{L} x \mathrm{R} y$ or $\mathrm{R} y \mathrm{~L} x, v=0$; or

(5) $\mathrm{L} x \mathrm{~L} x$ or $\mathrm{R} x \mathrm{R} y, v=0$.

Proof. Let there be $n$-syllables, such as $\mathrm{N} x, \mathrm{R} x, \mathrm{~L} y, \ldots$ Then by our formula 5.6 there are at least $n+1$ 's and at most $n-\frac{1}{2}$ 's. Hence the twisting $\# \geq n-n / 2-1=$ $n / 2-1$. Thus $n \leq 2$ and we have only a few cases to check. We list the arithmetic in the following table. Here $P$ means (\#L $y+\# \mathrm{R} x-\# \mathrm{~L} x-\# \mathrm{R} y$ ).

$$
\begin{aligned}
& n \quad \# x \quad \# y \text { RHS of formula } \quad \Rightarrow \text { 's conclusions } \\
& 110 \quad 1+0-1+P / 2 \quad P=0 \quad N x
\end{aligned}
$$

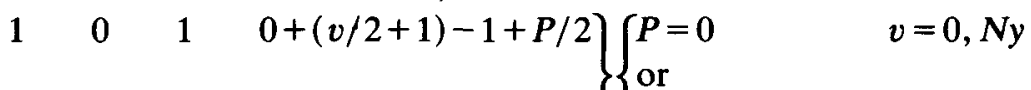

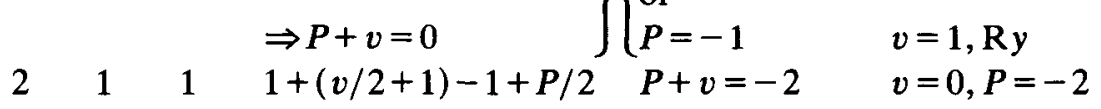

$$
\begin{aligned}
& \text { R } x \mathrm{~L} y \\
& 220 \quad 2+0-1+P / 2 \quad P=-2 \quad \text { Lx } 2 x \\
& 2020+2+v-1+P / 2 \quad v=0, P=-2 \quad \mathrm{R} y \mathrm{R} y, v=0
\end{aligned}
$$

Proposition 5.8. There must be an edge joint at a disk edge, that is to say, an edge joint at the side (or one of the two sides) at which $H=L(0, v)$ is not twisted.

Proof. We look at a component $T$ of $S^{2} \cap H$ which contains a point of the branch set. We proceed upward from such a point seeking an edge joint. We always proceed upwards making use of the $U$-strings whenever we encounter them according to the following prescription:

(5.8.1) In the case $H=L(0, v), v>0$, we always proceed along a $U$-string to its leftmost point and proceed upward from there. This way at any point of $T$ on the branch line we either:

(a) can proceed further upward using lines not previously encountered;

(b) have traced out a loop; then this loop has symbol $\mathrm{N} x$ as $H=L(0, v)$ and $v>0$; or

(c) encounter an edge joint; this must be on the left side of $H$ and hence we are done. 
Clearly case (a) can occur only finitely many times and case (c) only once. Thus it will complete the proof of (5.8.1) to show that case (b) cannot occur. To this end assume it does occur with $x$-arc $\xi$ joining points $a$ and $b$ on the branch line, where $a$ is to the left of $b$, and $U$-string $\gamma$ joining $a$ to $b$. Then $a$ is the left most end point of the $U$-string $\gamma$, so that the $x$-arcs proceeds upward from $a$, as in the following figure:
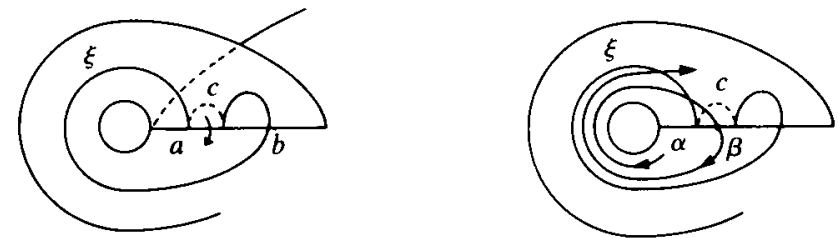

There is an arc of our knot guarding the leftmost of $\gamma$ 's $U$-joints, say at a point $c$. Then the arc of the knot leaving $c$ passes around to its next point $d$ on the branch set. Then $d$ is to the right of $c$ and thus to the right of $a$. Hence $\beta$ pierces $\xi$. But by the same token, the arc $\alpha$ of our knot arriving at $c$ must also pierce $\xi$, since it must begin to the left of $c$ and hence inside the loop $\Lambda$. This contradicts the no double entry lemma and completes the proof of 5.8.1.

(5.8.2) In the case $H=L(0,0)$, we use a mixed strategy. Having arrived at a $U$-string we proceed upward along its leftmost point only half the time, to wit, in case we arrived at the $U$-string along an $x$-line. However, if we arrive at a $U$-string on a $y$-line, we use the rightmost point for our upward process. This means in particular that the symbols $x \mathrm{R}$ and $y \mathrm{~L}$ never occur. This rules out case 4 of (5.7) and case 3 is ruled out as $v=0$. Thus whenever we are at a point of the branch we either:

(a) can proceed further;

$\left(b_{1}\right)$ trace out an exceptional loop with symbol $\mathrm{N} x$ or $\mathrm{N} y$;

$\left(b_{2}\right)$ trace out a loop with a symbol of type 5 in the list of (5.7); or

(c) arrive at an edge joint.

Now if case (c) occurs we are done as both of $H$ 's edges are disk edges. But by our previous argument in (5.8.1) we cannot have case $\left(b_{1}\right)$ so that it will suffice to show that case $\left(b_{2}\right)$ cannot occur.

To this end, assume we do encounter either $\mathrm{L} x \mathrm{~L} x$ or $\mathrm{R} y \mathrm{R} y(v=0)$. As these are alike, we will deal with $L x L x$. Let the rightmost point of our loop $\Lambda$ be $A$; note that from $A$ we must have a $U$-string going left, say ending with $B$ on the branch line. From $B$ we have an $x$-arc which arrives at a point, say $C$ on the branch set. We claim $C$ is between $A$ and $B$. To see this, assume not; then $C$ is to the left of $B$ as by our choice $A$ is the rightmost point. We have:

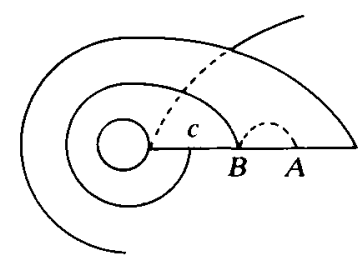


and we are trapped, as there is no way for the next $x$-arc to get back as far right as $A$. This leaves only the case that $C$ is between $B$ and $A$.

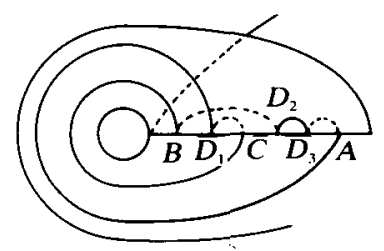

This is ruled out, for example, as follows: there must be an arc of $K$ guarding the $U$-joint at $C$. Let one such arc of $K$ enter along an $x$-line $\alpha$, at a point $E$. Then $E$ is between $D$ and $C$. Let $\beta$ be the next arc of the knot leaving $E$. Then $\beta$ crosses both of the $x$-lines of $\Lambda$, since $A$ and $C$ are to the right of $E$. But then $\alpha$ must also cross both of the $x$-lines of $\Lambda$ as

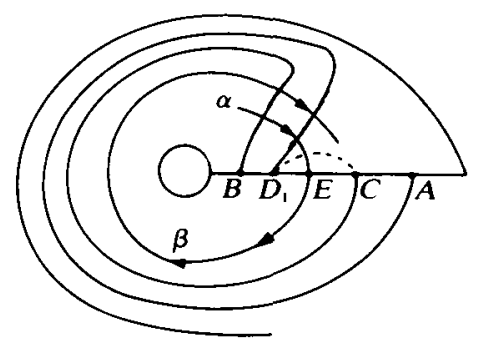

it begins on the branch set at a point to the left of $E$ and thus to the left of $A$ and $C$. But this makes a total of 4 points on $S^{2} \cap K$ though there are only two. This contradiction shows that case $\left(b_{2}\right)$ cannot occur and completes the proof of (5.8).

\section{Reduction at a disk edge}

Thus we have a $U$-string which ends at an edge-joint at a disk edge. There is the special case in which our $U$-string is a double edge joint, but the argument below works in this (rather trivial) case as well.

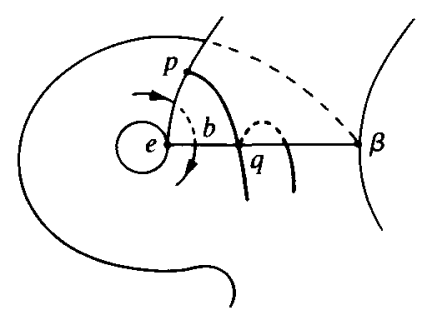

Case 1. Assume that our edge joint does not end on the disk edge itself but at $p$ on the outer edge. Let $q$ be the other end point of the edge joint ending at $p$ and let $e$ be the nearby end point of the branch set $\beta$. We know there is an arc of $K$ guarding the edge joint $p q$, say at a point $b \in \beta$. Now there are two kinds of arcs of the knot $K$ arriving at $e q$, those coming from another point of $e q$ and those arriving from afar. But as each arc of $K$ arriving at $e q$ from afar pierces $p q$ and as two pierces are impossible by $\S 1$, there is only one of this kind, arriving say, at $a$. Then $a$ is the point of $K \cap \beta$ closest to $e$. But now note that the entire section $\gamma$ of $K$ 
passing from $a$ to $b$ does so around the disk, with no other points of $K$ between $a$ and $b$. Hence the section $\gamma$ can be deleted without affecting the knot. But this contradicts the minimality of the knot, so that case 1 does not occur.

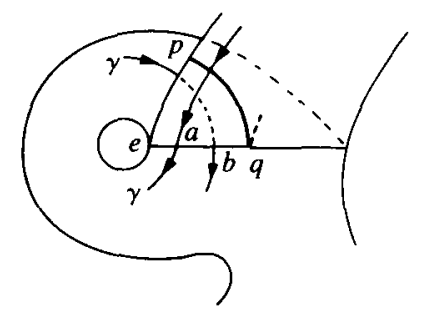

Case 2. The $U$-string ends on the disk of the disk edge.

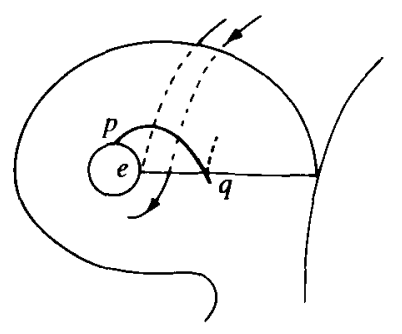

We again label our points $e, p, q$. Now since the component of $S^{2} \cap H$ containing $p q$ enters $D^{2}$, it must leave it again, after perhaps several passages through $D^{2}$.

There are three cases (see figure below). First it might leave $D^{2}$, finally on the upper part of the boundary. In this case it can only pass back to the branch set $\beta$. Secondly, it could leave $D^{2}$ on the lower arc of $\partial D^{2}$ but still pass back to the branch line $\beta$. Finally, it could leave on the lower arc and proceed to a boundary point of $H$. These three cases are drawn below. We have indicated beneath each one, the rather obvious deformation which decreases the number of segments without introducing branch points. Thus in any case our minimality assumption is contradicted. This completes the proof of the theorem.
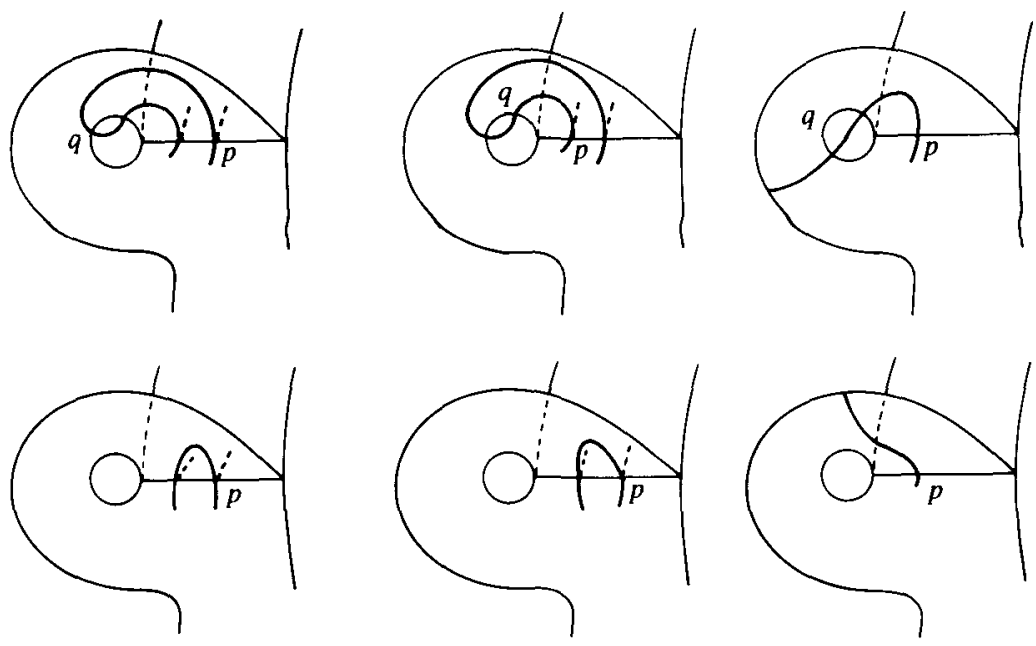


\section{Rogues Gallery}

This final section is devoted to showing why we were forced to make such a roundabout proof. Recall that up to $\S 5$ our analysis is good for all knot holders.

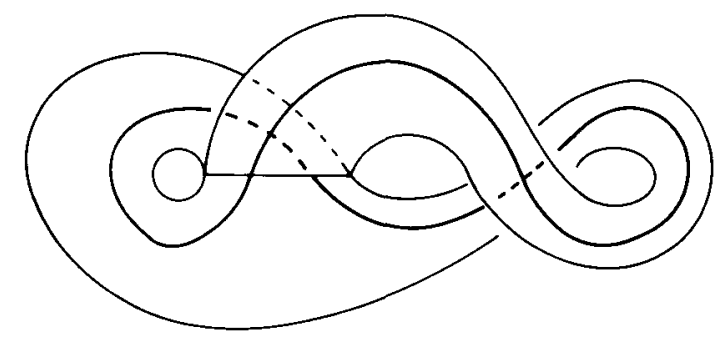

$\bar{L}(0,-2)$ with trivial loop $(x y)$.

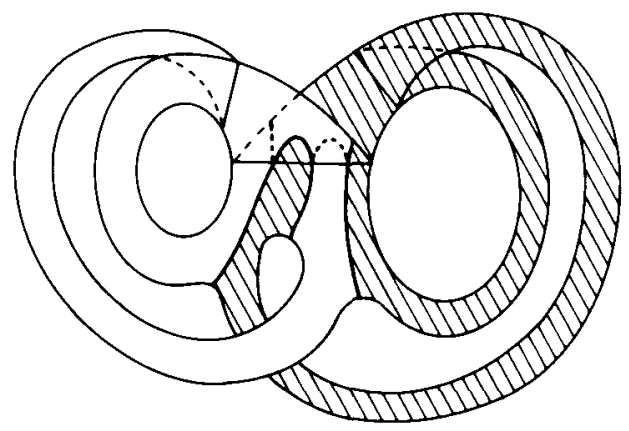

A composite knot holder $H^{\prime}$ with $S^{2} \cap H^{\prime}$ a tree.

At the beginning of $\S 5$ we used the fact that $H=L(0, v)$ or $\bar{L}(0, v)$ for $v \geq 0$. The argument given there would not apply for $v=-2$ as indicated by the loop corresponding to $x y$. It has a trivial tangent bundle in $H=\bar{L}(0,-2)$.

Secondly, we show by an example that one can have a tree with $U$-string top as intersection $S^{2} \cap H$. The knot-holder is not any of the $L(u, v)$ holders, but rather an ad hoc example. We have shaded the part of $H^{\prime}$ which is behind the separating sphere $S^{2}$. This $S^{2}$ can be thought of as a plane roughly parallel to the paper. The intersection $S^{2} \cap H^{\prime}$ is a 'tree' with a $U$-string top. Note that either side of $S^{2}$ contains a knot holder equivalent to $L(0,0)$. By an argument just like that of [2;6.1c] one can see that this last example contains all knots of $K_{1} \# K_{2}$, where both $K_{1}$ and $K_{2}$ are Lorenz knots.

As a last example, we give the simplest example we know of a knot holder with composite knots. In fact our $H^{\prime \prime}$ is a sub-knot-holder of the holder for the planetary orbits of the figure eight knot. See $\S 6$ of [2]. 


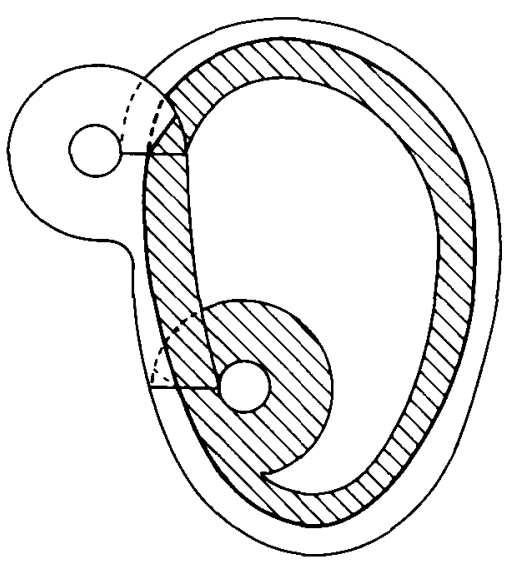

$H^{\prime \prime}$, a composite knot holder with $S^{2} \cap H^{\prime \prime}$ a loop plus 2 edge joints.

\section{REFERENCES}

[1] J. Birman \& R. Williams. Knotted periodic orbits I: Lórenz knots. Topology 22 (1983), 47-82.

[2] J. Birman \& R. Williams. Knotted periodic orbits II: Fibered knots. Low dimensional Topology, vol. 20, Contemporary Mathematics. Amer. Math. Soc: Providence. R.I. 1983.

[3] J. Guckenheimer. A strange, strange attractor. The Hopf Bifurcation (ed. Marsden and McCracken). Appl. Math. Sci., Springer-Verlag, 1976.

[4] J. Guckenheimer \& R. Williams. Structural stability of Lorenz attractors. Inst. Hautes Études Sci. Publ. Math. 50 (1979), 59-72.

[5] R. F. Williams. The structure of Lorenz attractors. Inst. Hautes Études Sci. Publ. Math. 50 (1979) 73-100. 